\title{
Corneal endothelial cell abnormalities in an early stage of the iridocorneal endothelial syndrome
}

\author{
W R Lee, G E Marshall, C M Kirkness
}

\begin{abstract}
A corneal disc, obtained from a 52-year-old woman suffering from an early stage of the iridocorneal endothelial syndrome (ICE), was investigated by various morphological techniques to analyse the structural variations in the endothelial cells and to identify the collagen types within the abnormal layer of Descemet's membrane. Scanning electron microscopy of the posterior corneal surface revealed a mosaic of (a) flat hexagonal cells resembling irregular but normal endothelial cells, and (b) rounded hexagonal (ICE) cells with numerous surface microvilli. Degenerative changes were present in each cell type, but were more common in the flat hexagonal cells which contained intracytoplasmic spaces. By transmission electron microscopy the flat hexagonal cells exhibited many of the features of normal endothelial cells in terms of organelles and intercellular attachments, but lateral invaginations were absent. The ICE cells differed in that the apical surface was covered by microvilli and the cytoplasm contained tonofilaments, which were also observed by light microscopic immunocytochemical staining. Most commonly, intercellular attachments were rudimentary in both types of cell and intercellular spaces were dilated, but desmosomes were sometimes prominent in the ICE cells where interdigitations were pronounced. In some sectors, the basal surface of the ICE cells was indented by deposition of clumps of fibrillar collagenous material. An immunocytochemical study of the abnormal posterior deposits localised type IV collagen to the amorphous matrix and collagen types III and $V$, but not type $I$, to the collagen fibril bundles. Mononuclear inflammatory cells were identified between the ICE cells in the monolayer. The evidence suggests that some of the flat hexagonal cells were undergoing a degenerative change while others were transforming into ICE cells.

(Br f Ophthalmol 1994; 78: 624-631)
\end{abstract}

The iridocorneal endothelial (ICE) syndrome is a rare non-familial unilateral disorder which progresses to glaucoma and is characterised clinically by a fine beaten silver appearance of all or part of the posterior corneal surface as seen by slit-lamp specular microscopy. ${ }^{12}$ By endothelial specular photomicroscopy, abnormal endothelial cells (ICE cells) have a characteristic appearance with a central highlight and a light peripheral surround..$^{2-5}$ The presence of ICE cells can herald (a) corneal oedema with a normal IOP and band-shaped keratopathy, (b) glaucoma caused by ICE cell migration across the trabecular meshwork (Chandler's syndrome), (c) glaucoma associated with iris atrophy (essential iris atrophy), or (d) iris melanocytic neoplasia (iris naevus syndrome). ${ }^{1-5}$ It is clear, however, that a spectrum of disease exists and many patients exhibit features of more than one of the classically described syndromes.

In pathological material, endothelial morphology has been inconsistent. By light microscopy, the endothelial cell population is depleted in some cases, particularly in Chandler's syndrome, ${ }^{6-8}$ and occasionally multilayered, although in the majority of specimens the cells have formed a degenerate monolayer. ${ }^{9}{ }^{10} \mathrm{By}$ scanning electron microscopy, the presence of abundant surface microvilli over hemispherical cells and cytoplasmic blebbing have been the most noteworthy feature of ICE cells. ${ }^{5-10}$ Flat hexagonal cells which varied considerably in size and shape have also been recorded as a mosaic or subpopulation on the posterior corneal surface. ${ }^{5911}$

By transmission electron microscopy ICE cells have been shown to have endothelial cell characteristics. $^{6-1012}$ By contrast, ICE cells have been shown to have epithelial characteristics with desmosomes ${ }^{61213}$ and intracytoplasmic tonofilaments. ${ }^{68101213}$

Descemet's membrane which normally contains two collagen types (IV and VIII) ${ }^{1+16}$ is thickened in ICE by the addition of a broad posterior collagenous layer (PCL) ${ }^{6-810-1217}$ occasionally containing wide banded collagen. ${ }^{6-917}$ Small foci of fibrillar deposits behind the posterior non-banded zone were reported in one example of the early stage of the disease. ${ }^{10} \mathrm{An}$ attempt to identify the collagen types in the PCL has not to our knowledge been attempted. In the present morphological study we describe the features of the endothelium in an early uncomplicated case of the ICE syndrome in which, with the application of immunogold immunocytochemistry, we have identified some of the constituents of the abnormal posterior collagenous layer which is secreted by the ICE cells.

Clinical history

A female patient (born 10 May 1941) attended
Professor W R Lee, Pathology Department, Western Infirmary, Glasgow G11 6NT Accepted for publication 8 February 1994 
the Tennent Institute in January 1993 with a unilateral decrease in visual acuity which was associated with some diurnal fluctuation in vision, the vision being worst in the morning and clearing slightly by mid-day. Such symptoms had persisted for 5 years. There was no family history of corneal disease. The referring ophthalmologist considered that the anterior chamber was shallow and that a few peripheral anterior synechiae were present. On examination the vision in the affected eye was reduced to $6 / 24$ with correction. There was stromal and epithelial corneal oedema and a distorted eccentric pupil with ectropion uveae. Fine endothelial detail was obscured by the oedema. No other features were distinguishable and in particular the other eye was entirely normal. Intraocular pressure in both eyes was within normal limits. Endothelial specular photomicroscopy (ESP) was performed with considerable difficulty because of the corneal oedema. In some areas, cells with central highlights were visible. Clinically this was felt to be ICE syndrome because of the unilaterality, the ESP appearance and the lack of any clinical features suggestive of posterior polymorphous dystrophy - namely, vesicles, snail track, and thickened Descemet's membrane. The small ectropion uveae was also felt to be consistent with the ICE syndrome.

A corneal graft was performed on 12 January 1993 and at follow up 9 months later the graft has remained clear and the intraocular pressure has remained normal.

\section{Materials and methods}

The keratoplasty disc measured $8 \mathrm{~mm}$ in diameter and the stroma was cloudy. The specimen was divided into four, and three parts were fixed in glutaraldehyde ( $2 \%$ ) for paraffin histology, scanning electron microscopy, and conventional transmission electron miscoscopy. The remaining part was fixed in a paraformaldehyde $(4 \%) / g l u t a r a l d e h y d e(0.5 \%)$ mixture for 2 hours at room temperature for immunogold immunocytochemistry. ${ }^{14}$ 15

Figure 1 Light micrographs of the posterior corneal surface. (a) Vacuoles (arrowheads) were a prominent feature in some areas of the endothelium. (b) In other sectors the cells were rounded and inflammatory cells (arrowheads) could be identified in the monolayer. (c) In the periodic acid Schiff stained paraffin section, Descemet's membrane (arrowheads) appears bilayered and the endothelial cells are vacuolated or attenuated. ( ( $a$ ) and $(b)$ Araldite toluidene blue, $\times 630 ;(c)$ paraffin $\times 750$.) (d) Some of the cells (corresponding to rounded cells) on the posterior surface stain positively with cytokeratin markers while other cells (corresponding to the flat vacuolated cells) are negative (CAM 5.2).
Paraffin sections were stained with haematoxylin and eosin, periodic acid Schiff, and Masson and were also used for an immunohistochemical study of cytokeratins in the epithelium and the endothelium. The following antibodies were applied - CAM 5.2, AE 1/3, NCL, and MNF. The tissue for transmission electron microscopy was processed through to Araldite and semithin sections were stained with toluidine blue. Ultrathin sections were examined in a Jeol 1200 EXII transmission electron microscope. The quadrant used for scanning electron microscopy was subject to critical point drying before gold coating and examination in a Jeol JSM 64000 scanning electron microscope. The block allocated for immunocytochemistry was processed for London resin (LR) white embedding. Details of the techniques for embedding, antibody labelling, and immunocytochemistry have been provided elsewhere..$^{14} 15$

\section{Results}

\section{LIGHT MICROSCOPY}

The epithelium was oedematous and the basement membrane was multilayered and infolded in parts: there was no evidence of a band keratopathy. Bowman's layer was intact and there were plentiful stromal keratocytes, but in one half of the cornea the lamellae appeared to be more widely dispersed owing to oedema. Descemet's membrane measured $6 \mu \mathrm{m}$ but there was evidence of multilayering in the PAS stained sections (Fig 1). The normal cuboidal endothelium was replaced either by vacuolated cells or rounded cells and some cells were remarkably attenuated (Figs 1a, 1b). Mononuclear inflammatory cells were present within the monolayer (Fig 1b). Consistent results were obtained with the immunohistochemical markers for cytokeratins. The rounded ICE cells stained positively while the flattened and vacuolated cells were weakly staining (Fig 1d). The epithelium stained positively for each of the antibodies used.

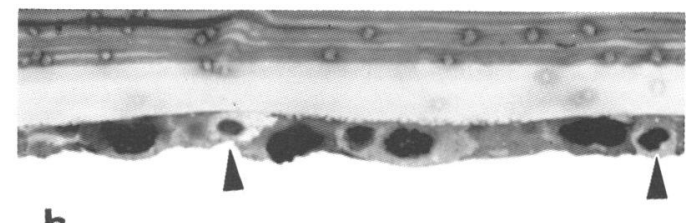

b

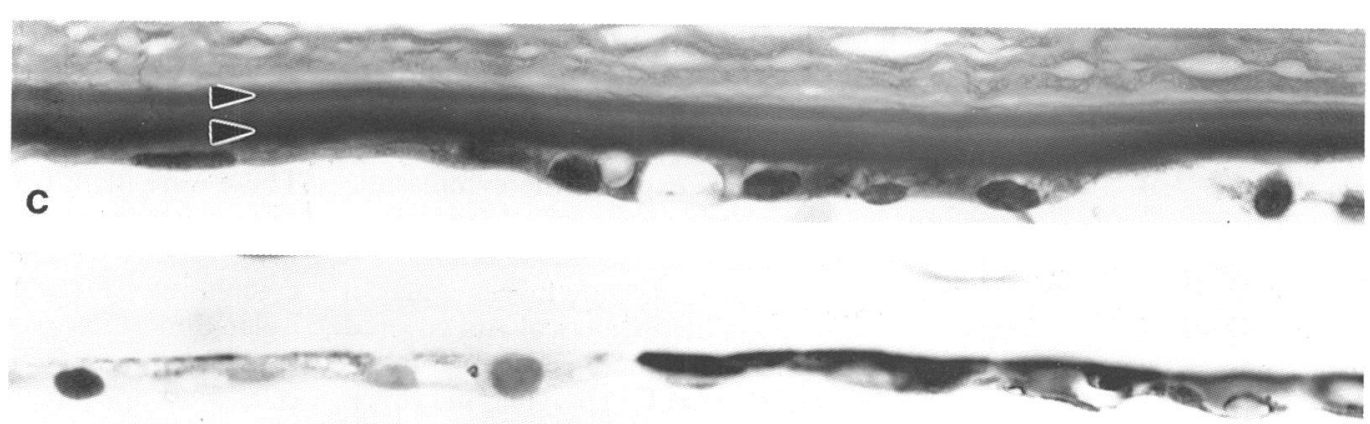


Figure 2 Features of the posterior corneal surface as seen by scanning electron microscopy. (a) Low power view of the flat hexagonal cells: note the degenerating cells (arrowheads). (b)

Higher power to contrast the surface of hexagonal cells with that of the ICE cells which are covered by microvilli: note cilia on the surface of the hexagonal cells (arrowheads). (c) The ICE cells sometimes showed widening of the intercellular spaces (arrow): note ruptured cyst in the hexagonal cells (arrowhead). (d) The ICE cells have prominent nuclear bulges and cilia (arrowheads). ((a) $\times 800$; (b) $\times 5000 ;(c) \times 1600 ;(d)$ $\times 1400$.)
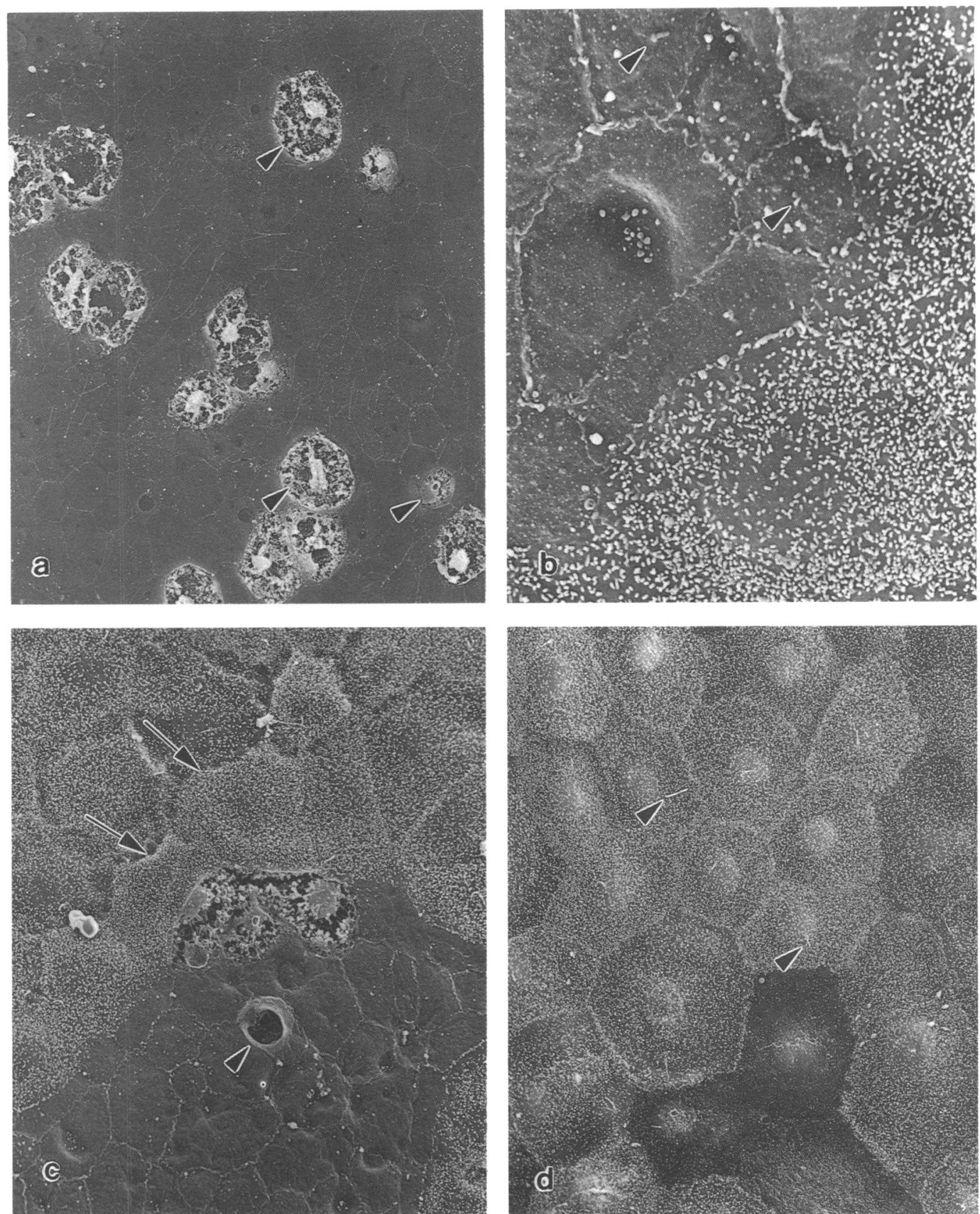

SCANNING ELECTRON MICROSCOPY

The appearance of the cells on the posterior corneal surface varied considerably. Small areas of flat cells resembled the normal hexagonal endothelium, but there was marked variation in shape and diameter (from 10 to $20 \mu \mathrm{m}$ ) of these cells (Fig 2). Cilia were present and the edges of the cells were lined by microvilli (Fig 2b). Ruptured cystic blebs exposed the cytoplasmic contents and extruded cytoplasmic debris lay on the surface of the adjacent hexagonal cells.

The majority of the cells were more regular in shape and size being hemispherical and covered by numerous microvilli: these cells are conventionally referred to as ICE cells. The microvilli were fewer in the central part of the cell, where there was a bulge and here a central cilium was easily identified. In other areas intercellular spaces were pronounced. Considerably fewer

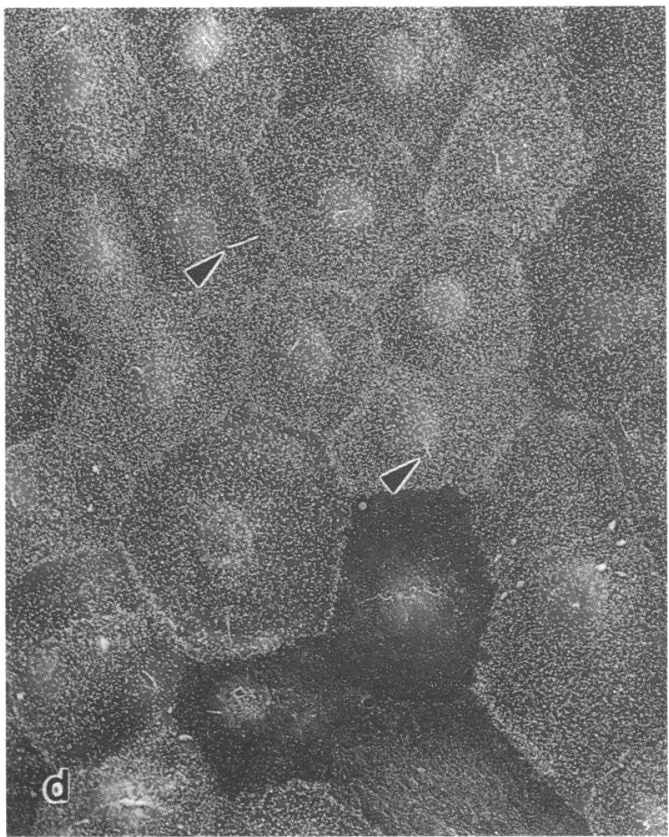

ruptured cysts were observed within hemispherical cell populations than among hexagonal cells. Binucleate cells were not seen.

\section{TRANSMISSION ELECTRON MICROSCOPY}

\section{Hexagonal cells}

These were identified as endothelial cells by the smooth posterior surface, typical mitochondria with tubular cristae, cilia, and a paucity of cytoplasmic filaments. Some cells contained isolated melanosomes (Fig 3) and areas of intracytoplasmic rarefaction and, in some groups of cells, what appeared to be large intracytoplasmic cysts were found. Intercellular lateral infoldings were not pronounced, but the junctions were those of the normal endothelium: occasionally the intercellular space was widened (Fig 3). 
Figure 3 (a) The hexagonal cells with a flat posterior surface contain numerous mitochondria and melanosomes: focal dilatations are present in the intercellular space (arrows) and within the cell (arrowhead). (b) A cilium within a cell (arrowhead) which contains

intracytoplasmic vacuoles. Note the close apposition between the cells and Descemet's membrane, and numerous mitochondria. ((a) and $(b) \times 7000$.

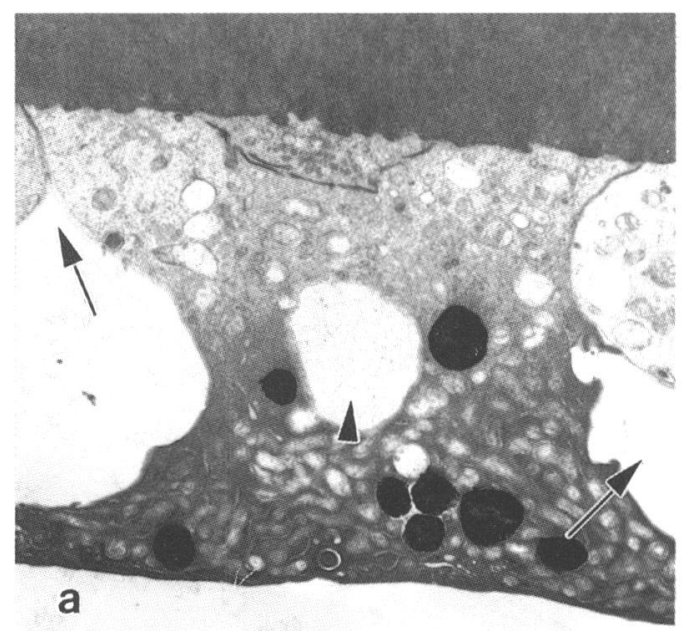

Hemispherical (ICE) cells

These were characterised by numerous microvilli on the posterior surface, abundant intracytoplasmic filaments $(80-100 \mathrm{~nm}$ diameter) (Fig 4) and mitochondria with lamellar cristae (Fig 5). Intercellular indentations were sometimes prominent and desmosomal atachments were an occasional finding. Some of the cells contained an isolated melanosome. Inflammatory cells (mainly lymphocytes and a few macrophages) were present within the monolayer. Descemet's membrane was thickened beneath the ICE cells by irregular deposition of clumps of striated fibrils embedded in an amorphous matrix: these nodules also projected into the cells (Fig 6). Wide banded collagen was absent.

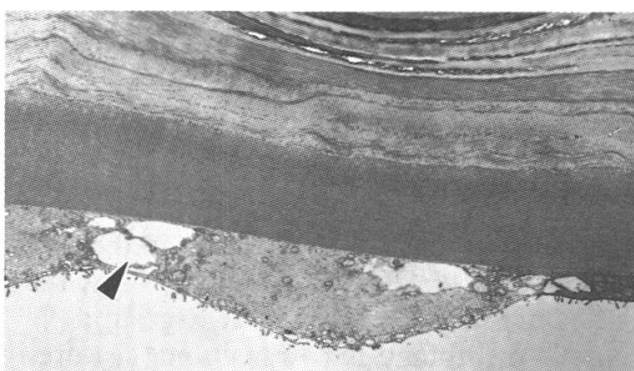

a

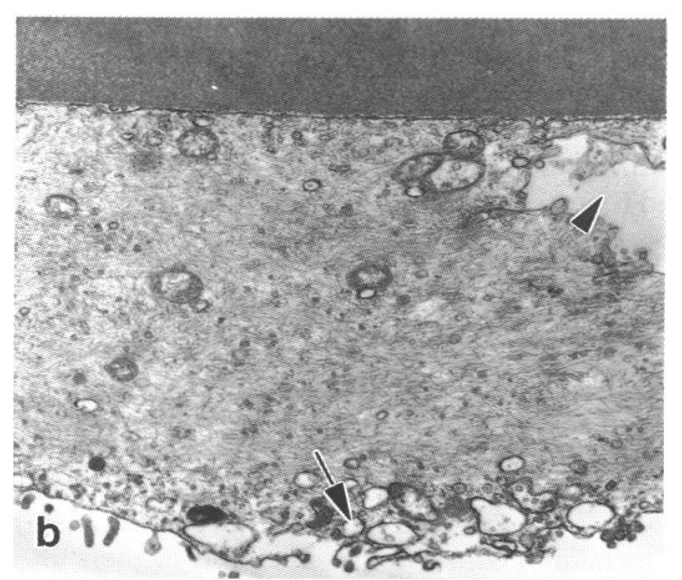

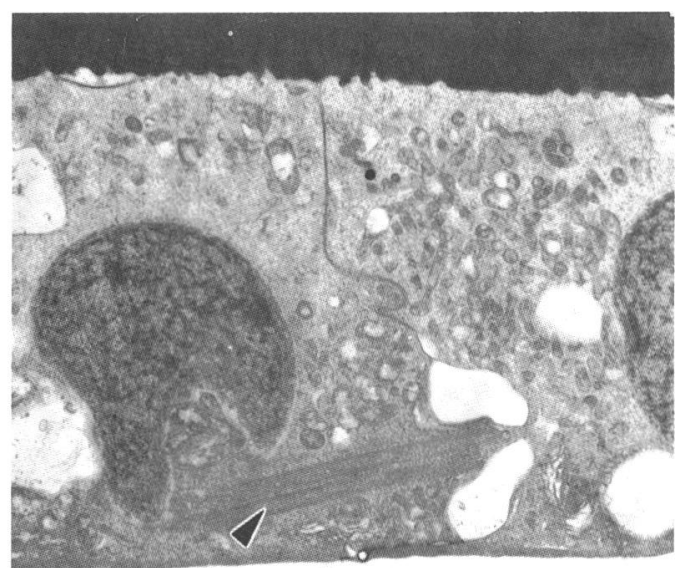

b

\section{IMMUNOGOLD IMMUNOCYTOCHEMISTRY}

Collagen types I, III, and V were localised to striated collagen fibrils in Bowman's layer and in the corneal stroma (Fig 7). Labelling for type IV collagen was present in the posterior non-banded zone of Descemet's membrane (Fig 8), but was absent from the basement membrane of the corneal epithelium. No labelling for type II collagen was seen in any of the regions studied. The localisation of collagen types $\mathrm{I}-\mathrm{V}$ in the present specimen, excepting Descemet's membrane (see below), was therefore identical to that seen in immunogold studies of aged human cornea. ${ }^{14-16}$

The accumulations of striated collagen fibrils which were observed between the ICE cells and Descemet's membrane were arranged either in whorls which invaginated the adluminal surface of the cells (Figs 8, 9) or in long thin bundles lying parallel to the endothelial surface (Fig 6). Both arrangements of fibrils exhibited intense labelling for collagen types III and V (Fig 9) and type IV was present in the enveloping amorphous matrix (Fig 8). Labelling for types I, II, and IV collagen was consistently absent from such fibril accumulations.

Immunogold particles were rarely observed in the rabbit antigoat immunogold control sections (omission of primary antibody) and were sparse in the normal goat serum control sections (that is, substitution of the primary antibody with non-immune serum from the same species in which the primary antibody was raised).

\section{Discussion}

Many of the cases reported as the ICE syndrome have been taken from patients suffering from abnormalities additional to those in the cornea. The value of the present case lies in the fact that it represents the disease at an early and uncomplicated stage.

In the present case, light microscopy was of value in confirmation of the clinical diagnosis of the ICE syndrome, because the flat vacuolated endothelial cells were easily distinguished from the humped ICE cells and immunocytochemistry identified cytokeratins in the latter cells. The focal positivity and minor variations we observed were also noted by Kramer et al, ${ }^{10}$ while more extensive staining was reported by Hirst et $a l^{12}$ : 
Figure 5 The

ultrastructural appearances of the ICE cells within the endothelium. (a) A low power view to show cells with prominent microvilli. (b) A mononuclear inflammatory cell is present within the monolayer (arrow). (c) In some areas desmosomal attachments (arrowheads) are present on the interdigitating cell membranes. (d) Dense contractile filaments are present within the cytoplasm of some ICE cells. ((a) $\times 2000 ;(b) \times 10000 ;(c)$ $\times 25000 ;(d) \times 100000$.

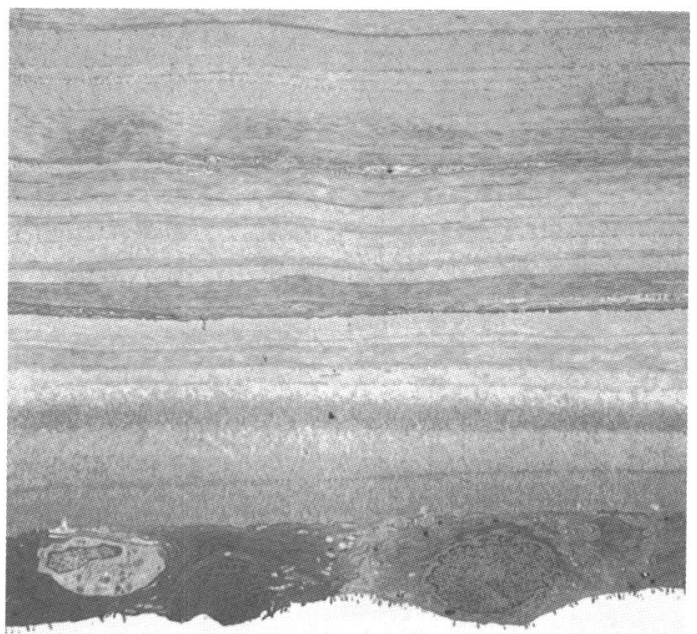

a

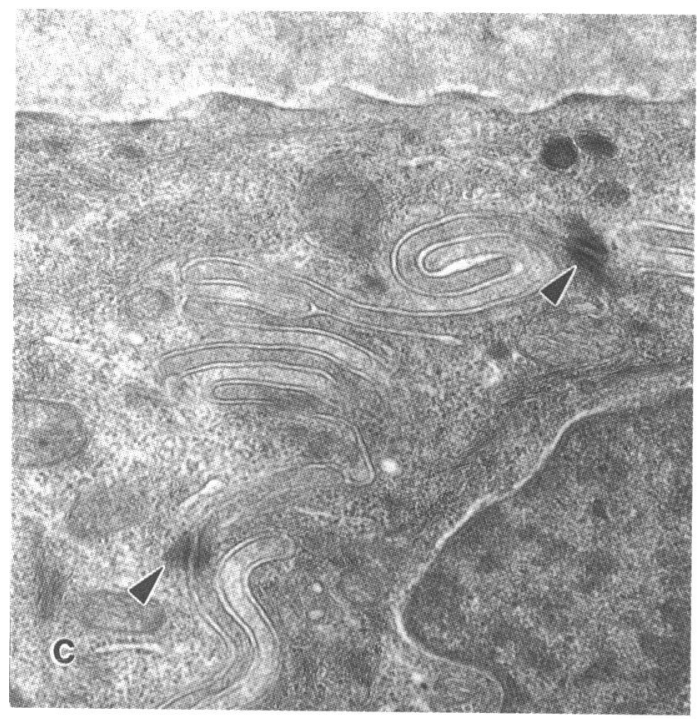

these differences may reflect the change from fla hexagonal cells to ICE cells as the disease progresses and at the end stage multilayering has been observed within the population..$^{91213}$ The presence of vacuolation in a distinct monolayer over a relatively thin Descemet's membrane is dissimilar to many of the cases described in the ICE literature, particularly in the reports of Chandler's syndrome in which Descemet's membrane was thickened and the cells were attenuated and/or duplicated. ${ }^{69911-13}$ Another discrepancy is that cystic blebbing (Fig 2) was more common in the hexagonal cells compared with the ICE cells in the present case, rather than in the ICE cells as has been reported by others. ${ }^{79}$ A thickness of $6 \mu \mathrm{m}$ of Descemet's membrane was a surprising feature in the present case since in all of the other published reports, Descemet's membrane has been markedly thickened. ${ }^{69-13} 17$ By light microscopy it was also possible to identify mononuclear inflammatory cells within the endothelium, but this feature, as these authors ${ }^{7-9}$ have stated, can only be regarded as supplemental to the diagnosis and is regarded as non-specific in other corneal disorders.

Scanning electron microscopy of the abnormal endothelium in the ICE syndrome provides a great deal of additional information concerning
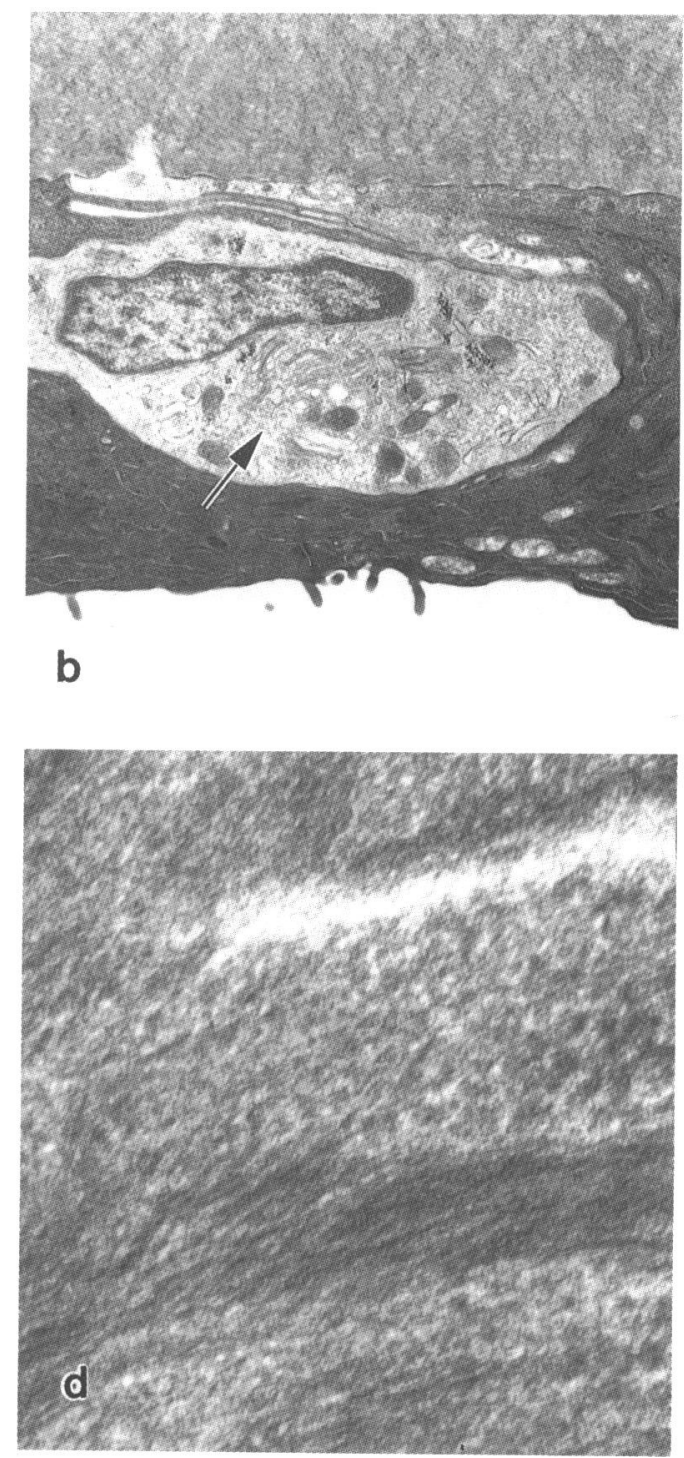

b

variations in cell morphology. The differences in appearance of the flat hexagonal cells and the ICE cells in the present case were very similar to those previously reported in various disorders in the ICE group, , ${ }^{51012}$ with some cells exhibiting transitional features between the flat and microvillous surfaces. Not all cases classified as ICE in the literature have demonstrated a continuous monolayer and illustrations of isolated cells with extended processes over an exposed Descemet's membrane have appeared. ${ }^{6813}$ This extreme stage of endothelial degeneration and atrophy appears to be associated with extension of the endothelium over the chamber angle onto the iris surface and therefore to massive depopulation of the corneal endothelium as in Chandler's syndrome. Depopulation is preceded by cell migration and this change is probably expressed as filopodial extension as seen by scanning electron microscopy, ${ }^{6-8}$ but this abnormality was not observed in other cases ${ }^{1012}$ or in the present case. This substantiates the impression that flat hexagonal cells are present in the endothelium at the earliest stage of the disease.

One of the striking features of the hemispherical ICE cells is that they cannot be classified as typical endothelial or epithelial cells on the basis of their ultrastructural features. While 
Figure 6 (a) Between the posterior collagenous layer and the ICE cells there is a layer of abnormal

extracellular matrix (arrowheads) shown at higher magnification in $(b)$ where there are bundles of collagen fibrils within an amorphous matrix. ((a) $\times 5000 ;(b) \times 20000$.)

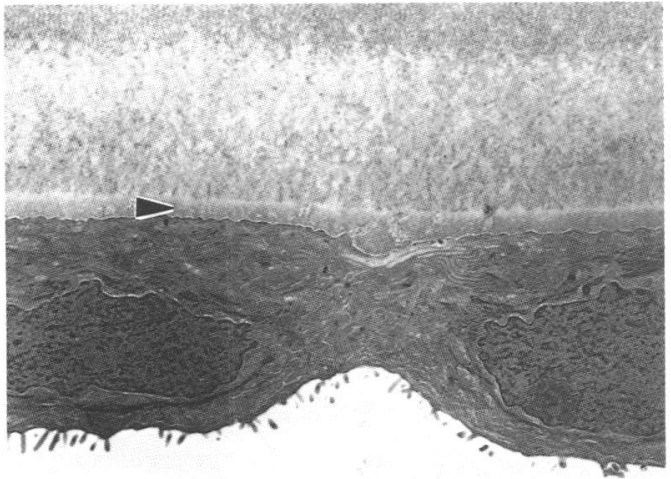

a

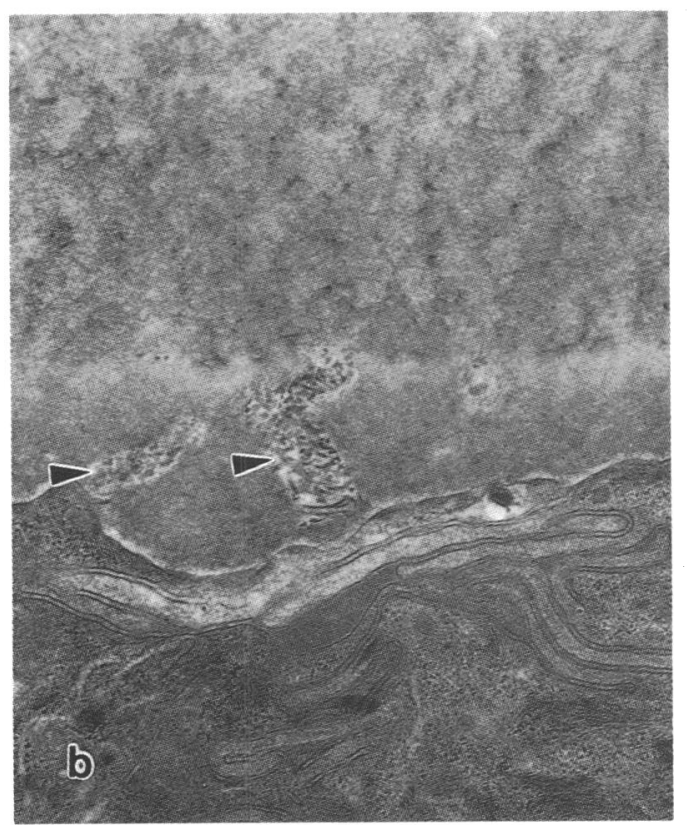

microvilli have been described in several papers ${ }^{1012}$ and cytoplasmic blebbing in the report by Alvarado et al, ${ }^{9}$ only four authors have emphasised the presence of intracytoplasmic tonofilaments ${ }^{61012}$ and desmosomal attachments, ${ }^{12} 13$ which characterise the cells as epithelial. Prominent tonofilaments and desmosomal attachments between multilayered cells favour a diagnosis of posterior polymorphous dystrophy (PPD), but for this unequivocal diagnosis an identifiable inheritance pattern and bilaterality are required. ${ }^{4}$ It is possible that there is an overlap between PPD and the ICE syndrome and there has been considerable interest and speculation in the metaplastic potential of the corneal endothelium, which may be based on its origin from the neural crest. ${ }^{18}$

The appearances of the dual cell population in the present case resembled in part the endothelial-type cells described by Alvarado $e t a l^{9}$ and the epithelial-type cells described by Kramer et al. ${ }^{10}$ The flat hexagonal cells did not have the typical features of endothelial cells in terms of intercellular boundaries, but contained the characteristic mitochondria and cilia. The epithelial-type cells exhibited intercellular invaginations, but were united by desmosomal attachments and lined internally by microvilli.
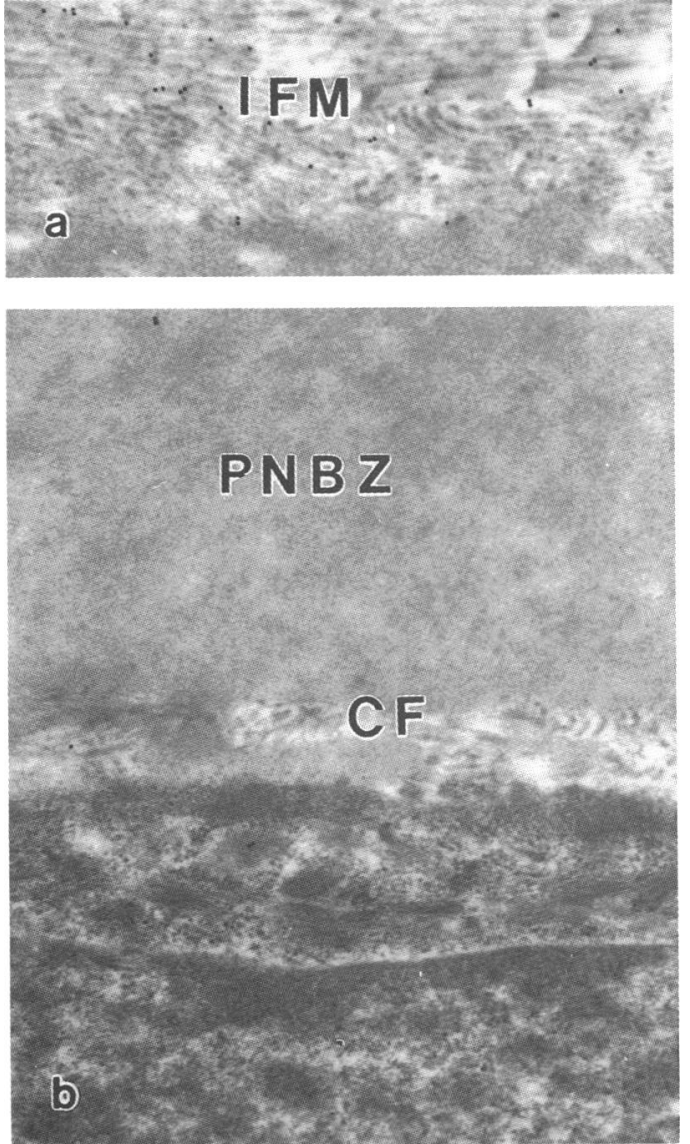

Figure 7 (a) The interfibrillar matrix (IFM) of the posterior stroma exhibits labelling for type I collagen. (b) Labelling for type I collagen is completely negative in the posterior nonbanded zone (PNBZ) and in a linear accumulation of collagen fibrils $(C F)$ at the endothelial face of Descemet's membrane. Note absence of label from endothelial cytoplasm and nucleus. $((a)$ and $(b)$ London resin white $\times 30000$.)

These differences are regarded by morphologists as of considerable importance in ascertaining the histogenetic origin of a cell population, but in the case of the corneal endothelium, derived from neural crest cells with a specific potential for metaplasia, the commonly accepted rules for an ectodermal or mesodermal origin do not apply. The mixture of cytoplasmic membrane characteristics observed in the cells on the posterior cornea suggests that there is a transformation from hexagonal cells to ICE cells and that the viability of the endothelial cells is lower than that of the ICE cells.

The presence of inter- and intracytoplasmic blebs in the flat hexagonal cells is strong evidence in favour of a functional failure to control water movement. It is tempting to suggest that the transformation to ICE cells with complex interdigitations is an attempt to restore the functional integrity of the posterior monolayer. This would be a stress response and it is reasonable to assume that synthesis of a new posterior collagenous layer is part of that response. The presence of microvilli would increase the surface area of the cell membrane, so that this may be an aberrant attempt to control water movement. The presence of leaking and non-leaking areas of cells may be relevant to the unpredictable nature of corneal oedema in the ICE syndrome. A decrease in water movement across the cornea in the ICE syndrome has been demonstrated by Bourne and 


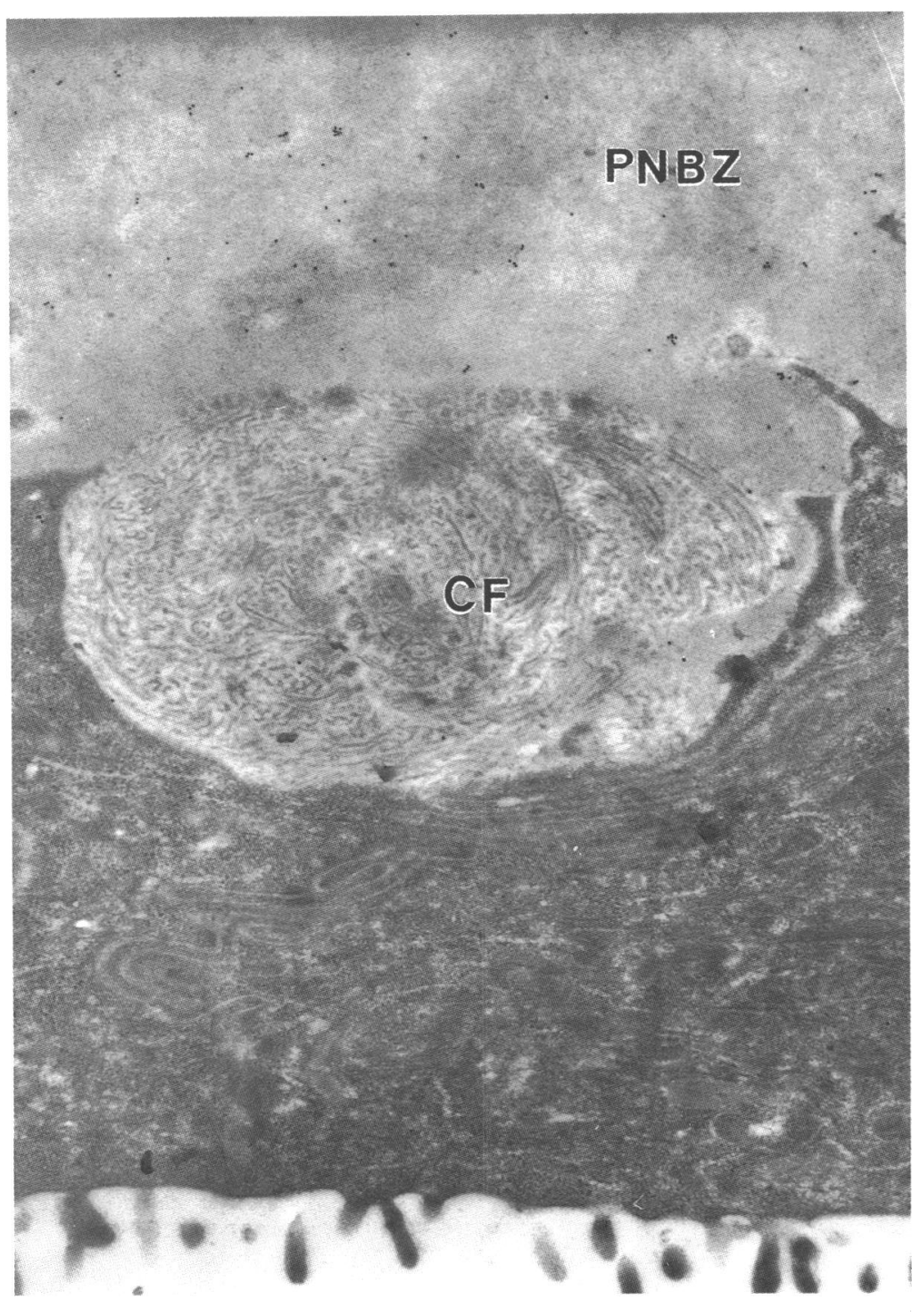

Figure 8 Type IV collagen labelling is present within the posterior non-banded zone (PNBZ) and over the amorphous material around a whorl of collagen filbrils (CF) that is invaginated within the endothelium and which is free from label.

(London resin white $\times 25000$.)

Figure 9 (a) Type III collagen labelling is intense and specifically localised to a bundle of collagen fibrils at the endothelial face of Descemet's membrane. The endothelial cytoplasm and posterior non-banded zone are free of label. (b) Type $V$ collagen labelling is also present over the posterior accumulation of collagen fibrils at the endothelial face of Descemet's membrane. (London resin white (a) $\times 30000 ;(b) \times 24000$.)
Brubaker ${ }^{19}$ using the iontophoresis technique with fluorescein. Bourne and Brubaker ${ }^{20}$ have shown more recently that the permeability of the cornea to fluorescein decreases with an increas-

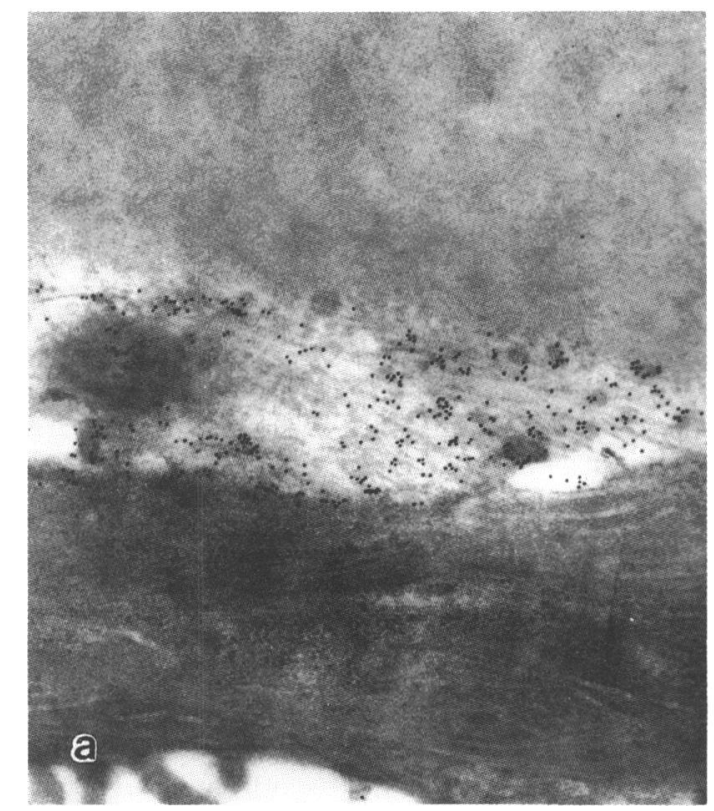

ing number of ICE cells and that ICE cells can regress in an individual patient with a permeability returning to normal. The corneas in such patients would not be available for morphological study, but the in vivo evidence suggests that in some ICE corneas there may be a transformational return to a cell monolayer which possesses the capacity to restrict water movement. If the concept is accepted that there is a capacity for recovery in some cases of ICE, the inflammatory basis for an unilateral disease as proposed by Alvarado et al ${ }^{9}$ has some basis.

In the present specimen lymphocytes were found without difficulty in the endothelium among both the ICE cells and the relatively normal hexagonal cells. Inflammatory cells within the endothelium are a striking abnormality and have been recorded in two publications $^{78}$ in addition to that of Alvarado et al. ${ }^{9}$ Rodrigues $e t a l^{7}$ suggested that an endotheliitis may be linked with a low grade iritis or that these cells are 'incidental' wandering cells. Certainly the presence of melanosomes within the hexagonal cells and the ICE cells suggests that there had been some breakdown of the pigmented cells of the iris.

The mononuclear inflammatory cells, lymphocytes, and macrophages may be responsible for the degenerative changes, vacuolation, and cell death described in the endothelial cell population in other reports ${ }^{89}$ and in the present case. In the present case it was apparent that the striated collagen fibres deposited presumably by the ICE cells contained types III and V and the amorphous matrix contained type IV. Type I collagen is not normally found in Descemet's membrane, ${ }^{14}$ type $\mathrm{V}$ collagen was located in the interfacial matrix between the stromal collagen and the anterior banded zone, ${ }^{14}{ }^{15}$ and type VIII collagen is located to the hexagonal lattice material of the anterior banded zone. ${ }^{16}$ It can therefore be concluded that the cells in the ICE syndrome were synthetically active but that the activity was aberrant in some aspects.

Cell culture experiments underline the latent ability of corneal endothelial cells to synthesise

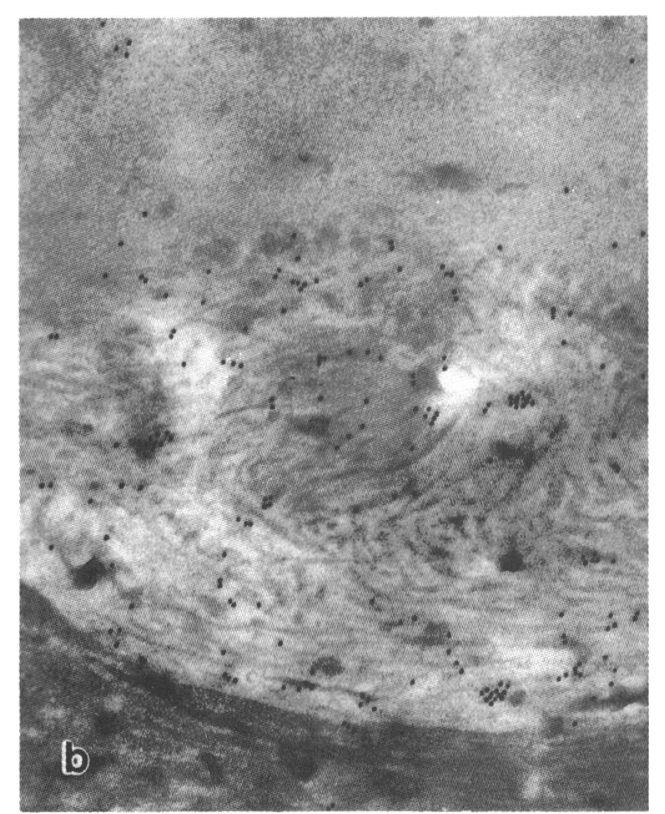


the number of collagen types that are normally not found in Descemet's membrane. ${ }^{16}$ Bovine corneal endothelial cells synthesise large amounts of types I, III, and V collagen when grown in cell culture (predominantly type III), whereas they synthesise types I and IV collagen when cultured on their Descemet's membrane. ${ }^{21}$ It would appear that expression of type I collagen synthesis has independent regulation from that of III and IV..$^{21}$ Therefore it is not surprising that ICE endothelial cells synthesised collagen types III and V without expression of type I collagen.

It is certainly relevant to note that polymorphonuclear leucocyte cells can modulate the synthetic activity of corneal endothelial cells by producing a corneal endothelial modulation factor (CEMF) that specifically transforms normal type IV collagen synthesising endothelial cells to type I synthesising cells. ${ }^{22}{ }^{23}$ Since the inflammatory cells observed in this study and other studies were probably lymphocytes or macrophages this raises the interesting possibility that mononuclear inflammatory cells can produce similar CEMFs which can stimulate the ICE cells to produce an abnormal matrix.

1 Wilson MC, Shields MB. A comparison of the clinical variations of the iridocorneal endothelial syndrome. Arch variations of the iridocorneal

2 Bourne WM. Partial corneal involvement in the iridocorneal endothelial syndrome. Am F Ophthalmol 1982; 94: 774-81.

3 Hirst LW, Quigley HA, Stark WJ, Shields MB. Specular microscopy of iridocorneal endothelial syndrome. $A m \mathcal{F}$ Ophthalmol 1980; 89: 11-21.

4 Laganowski HC, Sherrard ES, Kerr Muir MG, Buckley RJ Distinguishing features of the iridocorneal endothelial syndrome and posterior polymorphous dystrophy: value of specular microscopy. Brf Ophthalmol 1991; 75: 212-6.

5 Sherrard ES, Frangoulis MA, Kerr Muir MG. On the morphology of cells of the posterior cornea in the iridocorneal endothelial syndrome. Cornea 1991; 10: 233-43.

6 Richardson TM. Corneal decompensation in Chandler's syndrome. A scanning and transmission electron microsyndrome. A scanning and transmission elect
scopic study. Arch Ophthalmol 1979; 97: 2112-9.

7 Rodrigues MM, Stulting RD, Waring GO. Clinical, electron microscopic and immunohistochemical study of the corneal endothelium and Descemet's membrane in the irido-corneal endothelial syndrome. Am f Ophthalmol 1986; 101: 16-27.

8 Patel A, Kenyon KR, Hirst LW, Quigley HA, Stark WJ, Meyer RF, et al. Clinicopathologic features of Chandler's syndrome. Surv Ophthalmol 1983;27: 327-44.

9 Alvarado JG, Murphy CG, Maglio M, Hetherington J. Pathogenesis of Chandler's syndrome, essential iris atrophy and the Cogan-Reese syndrome. I. Alterations of the cornea endothelium. Invest Ophthalmol Vis Sci 1986; 27: 853-72.

10 Kramer TR, Grossniklaus HE, Vigneswaran N, Waring GO, Kozarsky A. Cytokeratin expression in corneal endothelium in the iridocorneal endothelial syndrome. Invest Ophthalmol Vis Sci 1992; 33: 3581-5.

11 Eagle RC, Shields JA. Iridocorneal endothelial syndrome with contralateral guttate endothelial dystrophy. Ophthalmology 1987; 94: 862-7.0.

12 Hirst LW, Green WR, Luckenbach M, de la Cruz Z, Stark WJ. Epithelial characteristics of the endothelium in
Chandler's syndrome. Invest Ophthalmol Vis $S c i$ 1983; 24: 603-11.

13 Quigley HA, Forster RF. Histopathology of the cornea and iris in Chandler's syndrome. Arch Ophthalmol 1978; 96: 1878-82.

14 Marshall GE, Konstas AG, Lee WR. Immunogold fine structural localisation of extracellular matrix components in aged human cornea. I Types I-IV collagen and laminin Graefes Arch Clin Exp Ophthalmol 1991; 229: 157-63.

15 Marshall GE, Konstas AG, Lee WR. Immunogold fine structural localisation of extracellular matrix components in aged human cornea. II Collagen types V and VI. Graefes Arch Clin Exp Ophthalmol 1991; 229: Graefes

16 Marshall GE, Konstas AGP, Lee WR. Collagens in ocular tissues. Brf Ophthalmol 1993; 77: 515-24.

17 Alvarado JA, Murphy CG, Juster RP, Hetherington J. Pathogenesis of Chandler's syndrome, essential iris atrophy and the Cogan-Reese syndrome. II. Estimated age at disease onset. Invest Ophthalmol Vis Sci 1986; 27: 873-82.

18 Bahn CF, Falls HF, Varley GA, Meyer RF, Edelhauser HF, Bourne WM. Classification of corneal endothelial disorders based on neural crest origin. Ophthalmology 1984; 91

19 Bourne WM, Brubaker RF. Decreased endothelial permeability in the iridocorneal endothelial syndrome.

20 Bourne WM, Brubaker RF. Progression and regression of partial corneal involvement in the iridocorneal endothelial syndrome. Am $\mathcal{F}$ Ophthalmol 1992; 114: 171-81.

21 Kay EP, Oh S. Modulation of type III collagen synthesis in bovine corneal endothelial cells. Invest Ophthalmol Vis $S c i$ 1988; 29: 200-7.

22 Kay EP, Ninomiya Y, Smith RE. Corneal endothelial modulation: a factor released by leukocytes induces basic fibroblastic growth factor that modulates cell shape and collagen. Invest Ophthalmol Vis Sci 1993; 34: 663-72.

23 Kay EP, Cheung CC, Chester JV, Nimni ME, Smith RE Type I collagen and fibronectin synthesis by retrocorneal fibrous membrane. Invest Ophthalmol Vis Sci 1982; 22: 200-12. 